\title{
Understanding the role of cross-language transfer of phonological awareness in emergent Hindi-English biliteracy acquisition
}

\author{
Priyanka Patel $^{1}$ (D) Nandini Chatterjee Singh ${ }^{2}$ D $\cdot$ Minna Torppa $^{1}$ (D
}

Accepted: 2 January 2022

(c) The Author(s) 2022

\begin{abstract}
This study examined within and cross-language relations, and specifically, the role of phonological awareness (PA) skills in reading among young Hindi-speaking children (L1) who were learning to read English (L2) in Delhi, India. Data was collected from 143 children in Grades 1 and 2 using measures validated for this population. The analyses examined the associations between L1 and L2 PA and decoding, both within and across the two languages. The results showed that PA skills within each language significantly predicted decoding in that language. Furthermore, there was evidence of cross-language transfer with Hindi PA significantly predicting English word reading even after controlling for English PA. English PA also significantly predicted Hindi decoding, however, these effects decreased once Hindi PA was added to the model. These findings emphasize the important role that both L1 and L2 PA plays in reading among emergent Hindi-English bilinguals. The theoretical and practical implications of these findings on literacy instruction in India are discussed.
\end{abstract}

Keywords Bilingual · Literacy · Hindi-English · Cross-language transfer · Phonological awareness · India

\section{Introduction}

Literacy has the power to transform lives, particularly among the world's most vulnerable populations. In countries with great linguistic diversity, however, the path to literacy acquisition is complex. India is home to over 300 million individuals who

Priyanka Patel

priyanka.v.patel@jyu.fi

1 Department of Teacher Education, University of Jyväskylä, Jyväskylä, Finland

2 UNESCO Mahatma Gandhi Institute of Education for Peace and Sustainable Development (MGIEP), New Delhi, India 
speak two or more of the approximately 462 languages (Simons \& Fennig, 2018). The educational policy for language instruction reflects the prevalence of multilingualism, requiring children to learn to read in three languages upon the completion of secondary school, of which two are typically Hindi and English (Joshi et al., 2017). However, there is great variation across the nation in the languages taught, their order, and the time at which they are introduced in school (see Menganathan, 2011). Seen as a language of opportunity, schools are increasingly offering English as a medium of instruction starting in Grade 1. Children in these schools are not only expected to learn to read in the instructional medium (e.g., English) but they are often simultaneously taught one of the Indic languages (e.g., Hindi), requiring the mastery of two distinct writing systems upon school entry (Joshi et al., 2017).

There exists a large and sound body of research on biliteracy acquisition. One of the major findings to emerge from this work has been that phonological skills in the first language (L1) can and do transfer to the second language (L2), as well as vice versa, facilitating reading in both languages (e.g. see Gottardo et al., 2021 for review). However, meta-analyses have revealed variations in these associations based on the languages examined, tasks used, age of the participants, and their instructional/linguistic experiences (Branum-Martin et al., 2012; Melby-Lervåg \& Lervåg, 2011), highlighting a need to study these associations in diverse populations and language pairs. Understanding these relations are critical as they may aid in the early identification of children at risk of reading difficulties and can help in the design of effective instruction and remediation.

Studies examining biliteracy acquisition among children in India are greatly limited. Those which have been conducted have largely ignored emergent readers, despite the fact that an increasing number of children are expected to learn to read in two languages upon school entry. Furthermore, existing studies have not examined bidirectional transfer despite evidence that in some languages, phonological processing skills in both L1 and L2 are related to reading in both languages (e.g., LaFrance $\&$ Gottardo, 2005). Finally, there are no studies to our knowledge which have specifically examined Hindi-English biliteracy despite these being two widely spoken languages in the country (Office of the Registrar General \& Census Commissioner India, 2011). In light of this, the present study aimed to fill some of the gaps in the existing literature base on biliteracy acquisition among children in India by examining the role of L1 and L2 PA on reading in a sample of emergent Hindi-English bilinguals.

\section{The role of phonological awareness in reading}

Reading, regardless of language, requires using the sounds of the spoken language to process the written script (e.g., Perfetti, 2003; Perfetti et al., 2005). More specifically, phonological awareness (PA), the ability to recognize, discriminate, blend, and manipulate the sounds in language, has long been identified as a critical skill for reading acquisition (Adams, 1990; Goswami \& Bryant, 1990; Wagner \& Torgesen, 1987). Prior to formal literacy instruction, PA develops through children's experiences with oral language (e.g., Carroll, 2001; Cooper et al., 2002). As children begin 
to engage in formal literacy instruction, their phonological sensitivity becomes more refined through instruction and increased exposure to language and print (Anthony \& Lonigan, 2004). PA is believed to develop hierarchically, with children in the early stages developing sensitivity to large phonological units (e.g., syllables and rhymes), and over time becoming increasingly more sensitive to smaller units (e.g., phonemes) (Goswami \& Bryant, 1990; Treiman \& Zukowski, 1991; Ziegler \& Goswami, 2005; see Pufpaff, 2009 for review). According to the Psycholinguistic Grain Size Theory, the rate at which these skills develop varies across languages as a result of orthographic differences which affects the availability of sounds in the spoken language, the consistency in how spoken language maps onto written language, and the granularity of the writing system (Goswami, 2010; Ziegler \& Goswami, 2005, 2006).

Many of the scripts used across the Indian subcontinent emerged from the Brahmi-derived writing system in which the basic unit of writing is the akshara (see Kandhadai \& Sproat, 2010 for a detailed description). While languages such as Hindi, Kannada, Oriya, Telugu, etc., have symbol sets that vary in appearance, they all share common psycholinguistic features (Nag, 2011). Each akshara represents either a vowel or a consonant-vowel syllable (e.g. क - $/ \mathrm{k} \Lambda /$, म - $/ \mathrm{m} \mathrm{N} /$ ). In their primary form, consonants are encoded with an inherent schwa vowel $(/ \mathrm{N} /)$ which is retained when the vowel appears in the word initial position. Otherwise, the schwa vowel can be replaced with other vowel sounds by placing vowel diacritics before, after, above, or below the consonant or syllable cluster (e.g., कू - /ku:/=क+ऊ; मी - / mi:/= म+ई) (Rao et al., 2021). Thus, although each distinct akshara represents sound at the syllable-level, each syllable consists of distinct phonemic units. As a result, akshara reading requires sensitivity to both syllable and phoneme-level information for decoding (Share \& Daniels, 2016; Vaid \& Gupta, 2002). Given the availability of distinct phoneme markers, the akshara orthographies are transparent. However, they are highly extensive with a symbol set of over 400 akshara and have great visuo-spatial complexity due to the non-linear arrangement of the symbols (Nag, 2007). Consequently, it has been found that akshara learning continues well into the elementary grades (e.g., Grades 4-5) (Nag, 2011).

\section{The role of phonological awareness in akshara reading}

Studies on akshara reading development have been reflective of the dual importance of PA, at both the syllable and phoneme-levels, in decoding (Mishra \& Stainthorp, 2007; Nag, 2007; Nag \& Snowling, 2012; Nakamura et al., 2018; Reddy \& Koda, 2013; Singh \& Sumathi, 2019). Singh and Sumathi (2019), examined reading development in 230 children across Grades 1-5 (ages 5-10) who were learning to read Hindi or Marathi. PA was assessed through a rhyme oddity task and a syllable replacement task in each language, and an average score was used for the analysis. PA significantly predicted word reading across grades and for children in Grades $3-5$, PA emerged as the strongest unique predictor of both word and pseudoword reading, reflecting the transparent nature of akshara-sound mapping. Unfortunately, due to the use of a composite score, it was not possible to tease apart the various contributions of rhyme versus syllable-level PA. 
In a study which examined children across Grades 1-4 it was seen that although both syllable and phoneme-level PA play a role in akshara decoding, syllable-level awareness plays a more dominant role, particularly in the early grades (Nag, 2007). Once children reached Grade 3, and had developed more advanced reading skills, phoneme awareness emerged as a slightly stronger predictor. Nevertheless, syllable awareness has been found to remain significant over time, including among older children (Grades 4-6). The salience of the syllable and its emphasis in reading instruction likely contributes to the stability of syllable-level awareness as a predictor of akshara reading (Nag \& Snowling, 2012). A more recent study which examined PA and reading in Kannada and Telugu across children in Grades 1-5 corroborated previous findings (Nakamura et al., 2018). While there were unique contributions of both syllable and phoneme awareness to word reading, children's syllable-level awareness seems to become sharpened over time and eventually subsumes phoneme-level awareness due to the saliency of the syllable in the akshara orthographies.

\section{Bilingual reading and cross-language transfer of phonological awareness skills}

PA, viewed as a universally required skill for reading, has received much attention in studies of biliteracy. A major finding to emerge from these studies is that L1 and L2 PA skills are highly correlated, and L1 and L2 PA can predict L1 and L2 reading (Gottardo et al., 2021; Melby-Lervåg \& Lervåg, 2011). This finding has since held true across a variety of language pairs such as English-Spanish, English-French, English-Korean, English-Chinese, English-Japanese, English-Kannada, and English-Kiswahili (e.g., Chen et al., 2010; Cisero \& Royer, 1995; Comeau et al., 1999; Durgunoğlu et al., 1993; Gottardo et al., 2001; Kim, 2009; Kuo et al., 2016; Reddy \& Koda, 2013; Wang et al., 2006; Wawire \& Kim, 2018). According to Koda's Transfer Facilitation Model (Koda, 2007, 2008), non-language specific aspects of PA, once developed in the L1, should be available for reading in the L2. As a result, we would expect PA in the two languages to be closely related. However, transfer would be expected to play a larger role when two languages share similar linguistic and orthographic properties which could be shared (Koda, 2008). Therefore, it is likely that different shared skills are transferred depending on the language pair.

Past studies have in fact revealed that there are differences in the facets of PA that transfer as a function of orthographic differences between languages. Bruck and Genesee (1995) found that English-speaking children in Grade 1 who were attending a French-immersion school had more advanced syllable-awareness skills in English than their monolingual peers, due to the saliency of the syllable in French. Chow et al. (2005) examined Chinese kindergarten students who were learning to read English and similarly found that Chinese syllable awareness significantly predicted English reading reflecting the role of the syllable as the basic phonological unit in Chinese. Interestingly, Chen et al. (2010) who examined Chinese children attending an intensive English program as compared to a regular English program found that children in the intensive group showed faster and higher growth on measures of Chinese rime and phonemic awareness, reflective of the phonological features of 
English. Thus, although PA is considered to be a language-universal construct, there is clear evidence that the pattern of transfer is reflective of the phonological features of the languages involved (see Branum-Martin et al., 2015), and that this is not only true for transfer from L1 to L2 but also from L2 to L1.

\section{Cross-language transfer of phonological awareness between the akshara languages and English}

Few studies have examined cross-language associations among akshara-English bilinguals. Mishra and Stainthorp (2007) conducted one of the first studies and examined 9-year-old (Grade 5) native Oriya speakers. The children attended schools which were either Oriya-medium $(n=48)$ or English-medium $(n=51)$ and had studied the other language as a subject starting at Grade 2. PA in English was assessed using the Test of Phonological Awareness (Hatcher et al., 1994) and an analogous test developed for Oriya. PA in English and Oriya were found to be highly correlated, however, the cross-language transfer of PA was not symmetrical between children in the two types of schools. For children who attended Oriya-medium school (Oriya is the first literacy language), PA in Oriya predicted decoding in both languages; a finding in line with previous studies of cross-language transfer. In addition, PA in English was found to predict word reading in English indicating that for children in Oriya-medium school, there is evidence of bidirectional transfer.

For children who were attending English-medium school (English is the first literacy language), PA in Oriya predicted pseudoword reading in Oriya and word reading in English and PA in English was found to only predict English decoding. Thus, while there was a role of transfer from the L1 to the L2, there was no bidirectional effect for children in English-medium school. Recognizing the impact of orthographic differences, the authors also examined the role of Oriya syllables versus Oriya phonemes. It was found that for those in Oriya-medium schools, awareness of the syllable made a significant unique contribution to Oriya decoding. Whereas for those in English-medium schools, it was awareness of the phoneme, and not the syllable, that predicted Oriya decoding. Authors concluded that this difference may be an effect of English phonemic awareness facilitating phonemic awareness in Oriya among the English-medium students. Unfortunately, these differential facets of PA were not examined across languages.

In a second study, Reddy and Koda (2013) specifically examined the effects of orthography-specific demands on decoding development among 10-14-year-olds $(n=52)$ who had received about four years of literacy instruction in Kannada and one year of literacy instruction in English. PA in both languages was assessed using analogous syllable-deletion and phoneme-deletion tasks. They found that while both syllable and phoneme-level awareness in Kannada contributed to Kannada decoding, only English phoneme-level awareness contributed to English decoding; reflecting the differential roles played by the syllable and phoneme in the two languages. Furthermore, it was seen that syllable and phoneme-level awareness in Kannada 
significantly contributed to English decoding, but this relationship was mediated by English phoneme awareness.

\section{Purpose of the study}

In the present study, we aimed to expand the current understanding of cross-language transfer of PA to include Grade 1 and Grade 2 Hindi speaking children who were attending an English-medium school. Specifically, we examined the strengths of the associations between the following:

1. PA in Hindi (L1) and decoding in Hindi (L1),

2. PA in English (L2) and decoding in English (L2),

3. PA in Hindi (L1) and decoding in English (L2),

4. PA in English (L2) and decoding in Hindi (L1).

We attempt to make three contributions to the gaps in the current research base on cross-language transfer of PA among akshara-English bilinguals. The first is by studying the role of PA in emergent readers. Previous studies conducted in India have all examined children in Grade 4 and above. While it is clear that at least among these older children with sufficiently developed literacy skills we see evidence of transfer, we do not know if the same is true for emergent readers. PA skill is well known to be the most powerful predictor of early reading (e.g., Melby-Lervåg et al., 2012). As students mature, however, PA and its relation to reading becomes less consistent (Scarborough, 2005). An increasing number of children are expected to learn to read in two languages upon school entry when literacy skills in both languages may still be rudimentary, thus it is important to explore patterns of transfer among these populations. Previous studies examining Chinese-English kindergarteners who were receiving literacy instruction in both languages, found a positive effect of L1 PA, specifically syllable awareness, on both L1 and L2 word reading (McBride-Chang et al., 2008). Although these young readers were still developing their PA in their L1, there still seems to be a meaningful role of L1 PA in L2 reading among this population.

The second contribution is the examination of cross-language transfer of PA at multiple grain sizes and bidirectionally. The examination of PA at multiple grain sizes is important as meta-analyses have indicated that composite tasks yield higher cross-language correlations than tasks only assessing PA at the syllable level, implying greater cross-language relations (Branum-Martin et al., 2012). The examination of PA at multiple levels will also allow us to better understand whether different phonological units have differential associations with word reading across the two languages. Although some previous studies in India have used multiple PA tasks (e.g. syllable-level and phoneme-level), these studies have not examined the transfer of these units both from the L1 to the L2 and vice versa. The final contribution is the addition of a new language pair to the research base. Although previous studies have examined akshara-English bilinguals, we believe 
it is important to replicate this work specifically examining Hindi-English bilinguals given the great amount of linguistic heterogeneity in India.

\section{Method}

\section{Participants}

The data for this study was collected as part of a larger study (see Patel et al., 2021) which included 143 students in Grades $1\left(n=70 ; M_{\text {age }}=5.73, S D=0.45\right)$ and 2 $\left(n=73 ; M_{\text {age }}=6.68, S D=0.47\right)$ who were attending an English-medium government school in Delhi, India. Prior to the start of the study, parents/guardians were invited to the school and taken through a consent form provided to them in both Hindi and English to ensure they were fully informed of their child's participation. The sample was made up of 43 boys and 27 girls from three Grade 1 classrooms and 31 boys and 42 girls from three Grade 2 classrooms.

The children attended school for approximately six hours per day, five days a week, during which all subject material was presented in English and children learned Hindi for about an hour per day. Hindi literacy instruction focused on the teaching of whole symbol blocks, with children learning the syllables that each akshara in the basic symbol register represents (see for e.g. Nag, 2011). English literacy instruction included some teaching of letter-sound correspondences and word families; however, teachers were heavily reliant on rote reading and copy-writing as is common in many English classrooms across India (see for e.g. Dutta \& Bala, 2012).

Information on children's out-of-school language use was collected through a questionnaire provided to the families. Out of 142 respondents, 118 families reported using only Hindi in the home, 19 families reported using both Hindi and English in the home, and 5 families reported using Hindi and/or another Indian language in the home. For children in India, another major source of language input is through private after school tutoring, which a majority of children attend (see Dongre \& Tewary, 2014 for a discussion on the impact of private tutoring in India). In our sample, 109 children (76.2\%) attended after school tutoring out of which 42 attended Hindi-medium centers, 6 attended English-medium centers, and 60 attended centers where both Hindi and English were used.

\section{Measures}

Children's literacy skills were assessed using the Dyslexia Assessment Battery from the Dyslexia Assessment for Languages of India (DALI-DAB) (Singh, 2015). The DALI-DAB is one of the first standardized and validated tools for the assessment of literacy skills in both Hindi and English, designed along similar lines as the Phonological Assessment Battery (PhAB; Frederickson et al., 1997), specifically for use in India (see Rao et al., 2021 for validation details). The DALI contains a separate 
battery for children in Grades 1-2 and Grades 3-5 to ensure that the tasks are developmentally appropriate, and the tasks were developed keeping in mind the orthographic properties of the respective language. The data used for this study was collected using the semantic fluency, rapid automatized naming (RAN), phonological awareness, and word reading subtests from the Grade 1-2 battery of the DALI in both Hindi and English. An additional word reading task as well as a pseudoword reading task developed for this population, but not a part of the DALI, were also used (Cherodath \& Singh, 2015).

\section{Semantic fluency}

In the semantic fluency task, participants were given $30 \mathrm{~s}$ to name objects in two given categories (i.e., animals and vegetables for Hindi and fruits and vegetables for English). For each language, a sum score of the correctly named items across both categories was used.

\section{Rapid automatized naming (RAN)}

RAN was measured using an object naming task from the DALI in which participants were presented with line drawings of five common objects: shoe, flower, house, chair, and key for Hindi and cup, bird, clock, van, and pencil for English, which were randomly arranged in 5 rows of 10 objects each. The participants were asked to name all of the objects in order as quickly as possible and the time taken was measured in seconds.

\section{Phonological awareness (PA)}

Hindi syllable replacement In line with the orthographic properties of Hindi in which each akshara represents a syllable unit, PA in Hindi was assessed at the syllable-level using a syllable (akshara) replacement task. The task consisted of two training trials and 10 experimental trials. In each trial, children were orally presented with a word and asked to replace the initial akshara (syllable unit) with a given akshara. (e.g., घर (Gar) with $\langle$ स $>$, response $=$ सर (Sar)). A score of one was given for every correctly formed new word. Cronbach's alpha reliability was 0.89 .

English phoneme replacement PA in English was assessed as at the phoneme-level through a phoneme replacement task. The task consisted of two training trials and 10 experimental trials. In each trial, children were orally presented with a word and asked to replace the initial phoneme with a given phoneme (e.g., replace /c/ in cot with $/ g /$, response=got). A score of one was given for every correctly reproduced word. Cronbach's alpha reliability was 0.89 .

Rhyme oddity PA in both languages was also assessed using a rhyme oddity task consisting of two training trials and 12 experimental trials. In each trial, children were orally presented with three words out of which they were asked to identify the 
rhyming pair (e.g., Hindi: नाम (naam) - काम (kaam) - नील (neel), English: madehide-fade). A score of one was given for every correct response. Cronbach's alpha reliability for the Hindi and English rhyme oddity task was 0.80 and 0.83 , respectively.

\section{Word reading}

Hindi word reading was measured using two separate word lists. The first list was from the Hindi DALI and consisted of 25 words collated from 1st and 2nd grade textbooks, arranged in order of increasing difficulty. The second word reading list consisted of 20 items taken from Grade 1-3 textbooks (Cherodath \& Singh, 2015). Children were instructed to read all items and a score of one was given for every correctly read word.

English word reading was also measured using two separate word lists. The first word reading list was taken from the English DALI and consisted of 25 words collated from 1st and 2nd grade textbooks arranged in order of increasing difficulty. The second word reading list consisted of 20 items taken from Grade 1-3 textbooks (Cherodath \& Singh, 2015). Children were instructed to read all items and a score of one was given for every correctly read word.

In both Hindi and English, the two word readings lists were found to be highly correlated (Hindi: $r=0.93$, English: $r=0.86$ ), and therefore the averages of the two scores in each language were used for analysis. Cronbach's alpha reliability of the combined word reading list was 0.99 for both Hindi and English.

\section{Pseudoword reading}

Pseudoword reading was assessed in both languages using a pseudoword list developed by Cherodath and Singh (2015). The English list consisted of 20 items in which a single letter was replaced in a real word to create a legally pronounceable string in English. Similarly, the Hindi list consisted of 20 items in which a single akshara was replaced to create a legally pronounceable string in Hindi. A score of one was given for every correctly read pseudoword. Cronbach's alpha reliability for the Hindi and English pseudoword reading tasks was 0.94 and 0.91, respectively.

\section{Procedure}

Children were brought into a quiet room within the school where they were assessed one-on-one across two sessions, one for Hindi and the other for English, each lasting about $20 \mathrm{~min}$. The assessment sessions were conducted classby-class, either in Hindi first or English first. Once all six classrooms had been assessed, children were brought back and assessed in the other language, thereby ensuring that children had a sufficient break between the two assessment sessions. 
The Hindi tasks were administered by three research assistants who were bilingual English-Hindi speakers. The English tasks were administered by the lead researcher who is a native English speaker and the Hindi-English bilingual research assistants. All of the assessors had a minimum of a bachelor's degree and prior experience working with children. Prior to the start of the study, a training session was held with the assessors on the administration and scoring of the data collection tools to ensure full understanding and consistency.

\section{Data analysis}

The data was analyzed using IBM SPSS and R Studio. First, a pairwise $t$-test was conducted to compare children's performance on the Hindi and English measures. Hierarchical linear regression was then employed to examine the unique effects of the various sub-skills in predicting word and pseudoword reading within and across the two languages. In hierarchical regression, predictor variables are added in steps, thereby allowing us to examine the unique effect of each variable(s) over and above the previously entered variable(s). In other words, this method allows us to examine the effect of a variable(s) after controlling for the effects of other variable(s). Effect size was measured using Cohen's $f^{2}$ according to which a value of 0.02 is a small effect, a value of 0.15 is a medium effect, and a value of 0.35 is a large effect (Cohen, 1992).

In all the models, children's age, and performance on the semantic fluency and rapid automatized naming (RAN) tasks were entered into the model first, allowing us to control for the variance emerging from differences in age and children's oral language/naming skills. Semantic fluency, while typically used as a measure of executive function, is also affected by vocabulary (Kavé, 2006). RAN, which assesses the ability to rapidly access names from visual symbols, has long been identified as a strong predictor of reading across a variety of languages including English (see for e.g., Compton, 2003) and Kannada (Nag \& Snowling, 2012). The phonological awareness measures were then entered into the model in alternating blocks, allowing us to examine the unique contribution of each phonological awareness task over and above the other.

Commonality analysis was conducted to determine the variance contributed by the PA tasks within and across the languages. Commonality analysis allows us to decompose the regression effect into unique and common effects, allowing a more accurate interpretation of the results, particularly when there is multicollinearity among the predictor variables (Kraha et al., 2012; Nimon, 2010). The analysis was conducted using the 'yhat' package (Nimon et al., 2020) in R Studio. We also calculated the squared structure coefficients allowing us to examine the percentage of the regression effect explained by each predictor (Thompson, 2006). 
Table 1 Descriptive statistics and paired samples t-test comparison of the Hindi and English measures

\begin{tabular}{|c|c|c|c|c|c|c|c|}
\hline & \multicolumn{3}{|c|}{ Hindi } & \multicolumn{3}{|c|}{ English } & \multirow[t]{2}{*}{$t(142)$} \\
\hline & Min & $\operatorname{Max}$ & $M(S D)$ & Min & Max & $M(S D)$ & \\
\hline Semantic fluency & 1 & 21 & $9.64(3.60)$ & 0 & 16 & $7.26(3.56)$ & $7.52 * * *$ \\
\hline RAN & 35 & 127 & $66.78(18.87)$ & 43 & 161 & $83.33(30.25)$ & $-7.64 * * *$ \\
\hline Rhyme oddity & 0 & 12 & $7.57(3.07)$ & 0 & 12 & $5.06(3.37)$ & $9.81 * * *$ \\
\hline $\begin{array}{l}\text { H Sylla- } \\
\text { ble replacement/E } \\
\text { Phoneme replace- } \\
\text { ment }\end{array}$ & 0 & 10 & $6.06(3.63)$ & 0 & 10 & $4.26(3.30)$ & $6.95 * * *$ \\
\hline Word reading & 0 & 22.5 & $17.64(5.78)$ & 0 & 22.5 & $10.83(6.49)$ & $18.29 * * *$ \\
\hline Pseudoword reading & 0 & 20 & $14.04(6.22)$ & 0 & 19 & $5.47(5.32)$ & $18.57 * * *$ \\
\hline
\end{tabular}

$H$ Hindi, E English

$* * * p<.001$

\section{Results}

Descriptive statistics for the Hindi and English tasks and paired sample $t$-test results comparing children's skills in the two languages are reported in Table 1. Results showed that children performed significantly better on the Hindi tasks, reflecting that Hindi was the more dominant language in this sample. Partial correlations, controlling for the effect of age, are presented in Table 2. The correlations between PA in Hindi and English, decoding in Hindi and English, PA in Hindi and decoding in English, and PA in English and decoding in Hindi were all moderate to high and significant.

\section{Predicting Hindi decoding with Hindi PA}

First, hierarchical regression analysis was used to examine the contribution of the Hindi PA tasks to Hindi word and pseudoword reading (see Tables 3, 4 respectively). Age was entered into the model first, followed by Hindi semantic fluency and Hindi RAN as covariates, and finally the Hindi PA tasks were entered in alternating order (Steps 3 and 4) to examine the contribution of each over and above the other.

In predicting Hindi word reading (see Table 3), the total variance explained by the model was $42 \%$, with the Hindi PA tasks explaining $17 \%$ of the variance over and above the covariates. Both Hindi rhyme oddity and syllable replacement were found to be uniquely associated with Hindi word reading. Results of the commonality analysis indicated that Hindi rhyme uniquely explained 3\% of the variance in Hindi word reading and made $69 \%$ of the contribution to the final regression model. Hindi syllable replacement emerged as the strongest unique predictor of Hindi word reading, explaining $6 \%$ of the variance and making $67 \%$ of the contribution to the final regression model (Table 3, Step 4). 


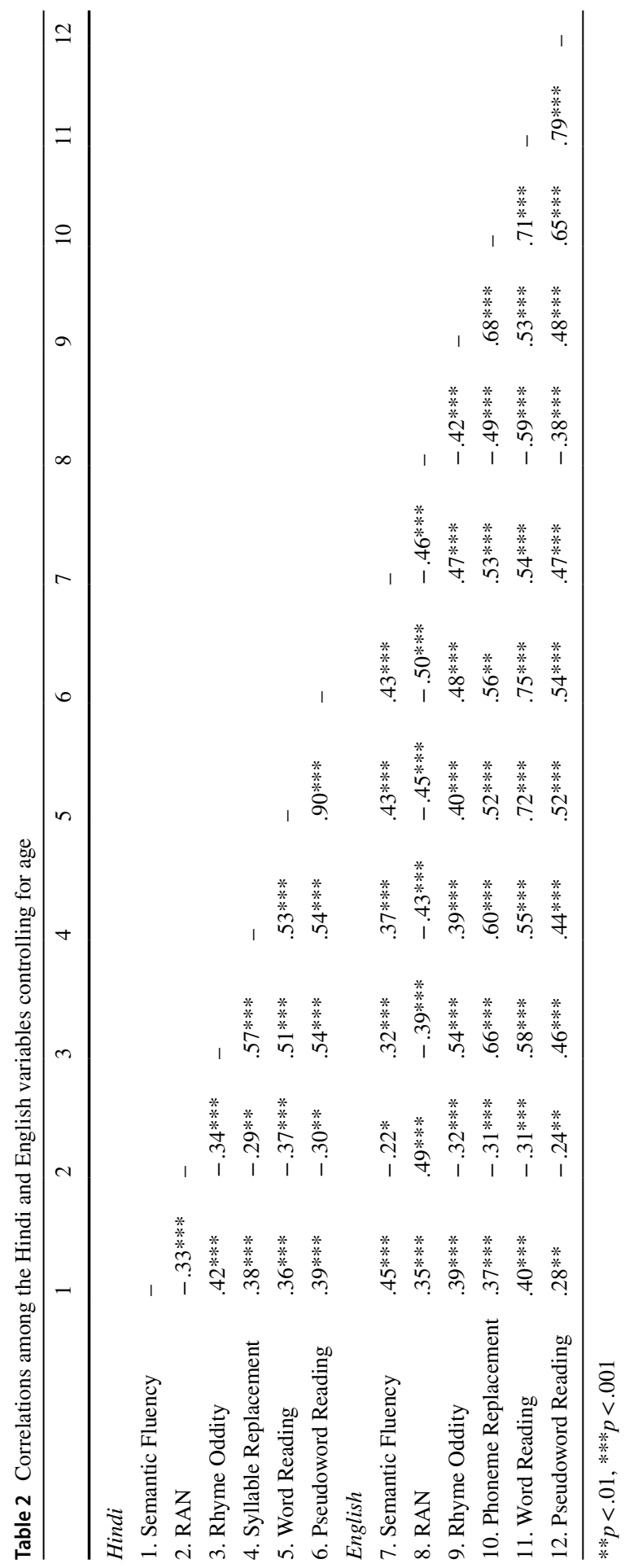




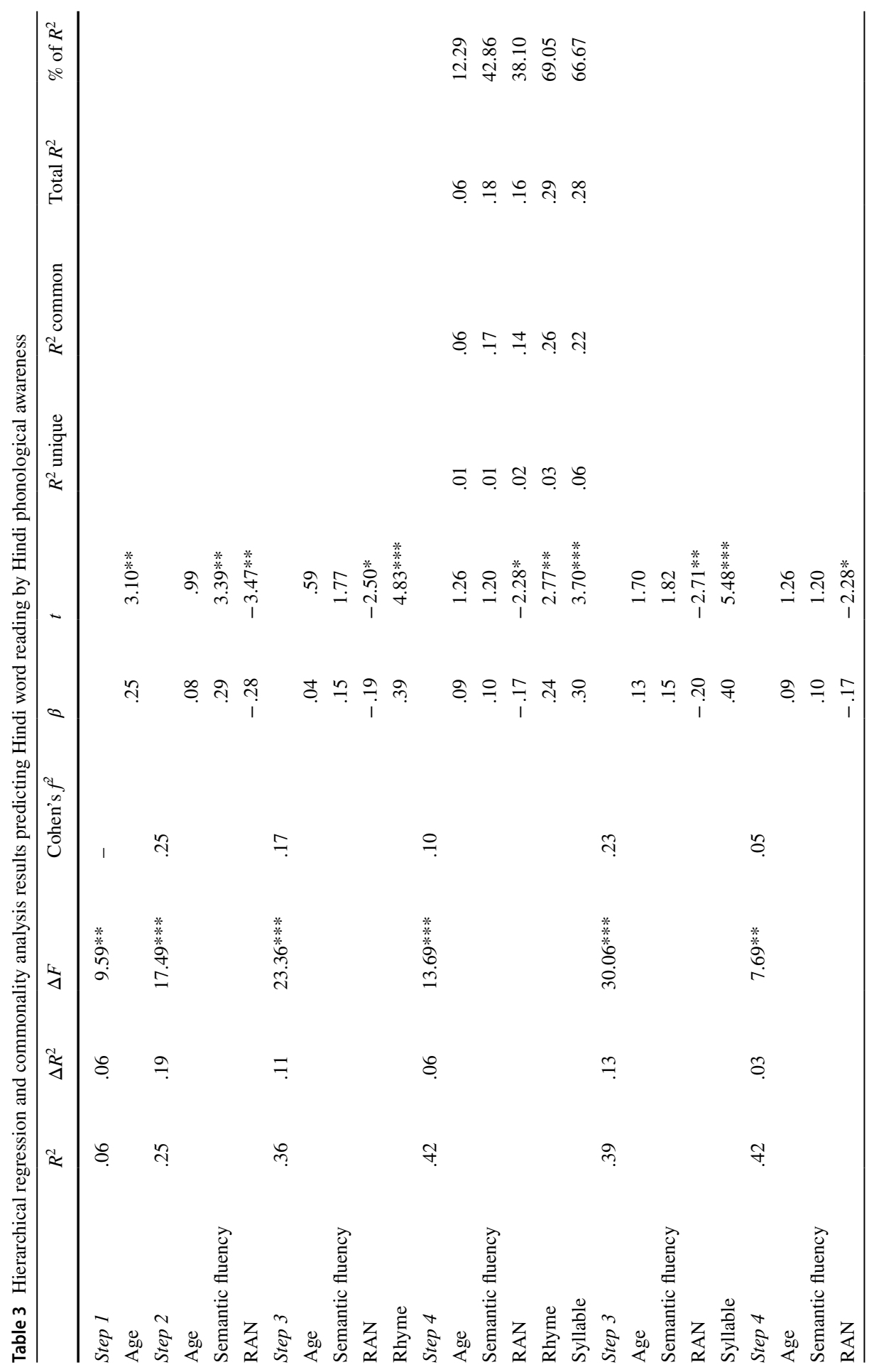




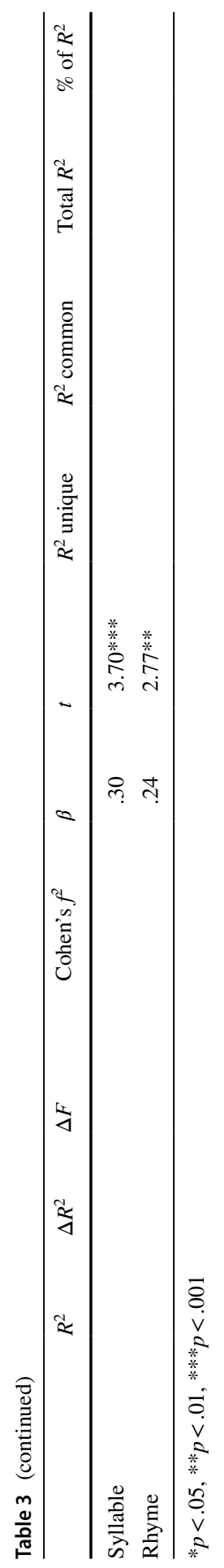

를 Springer 


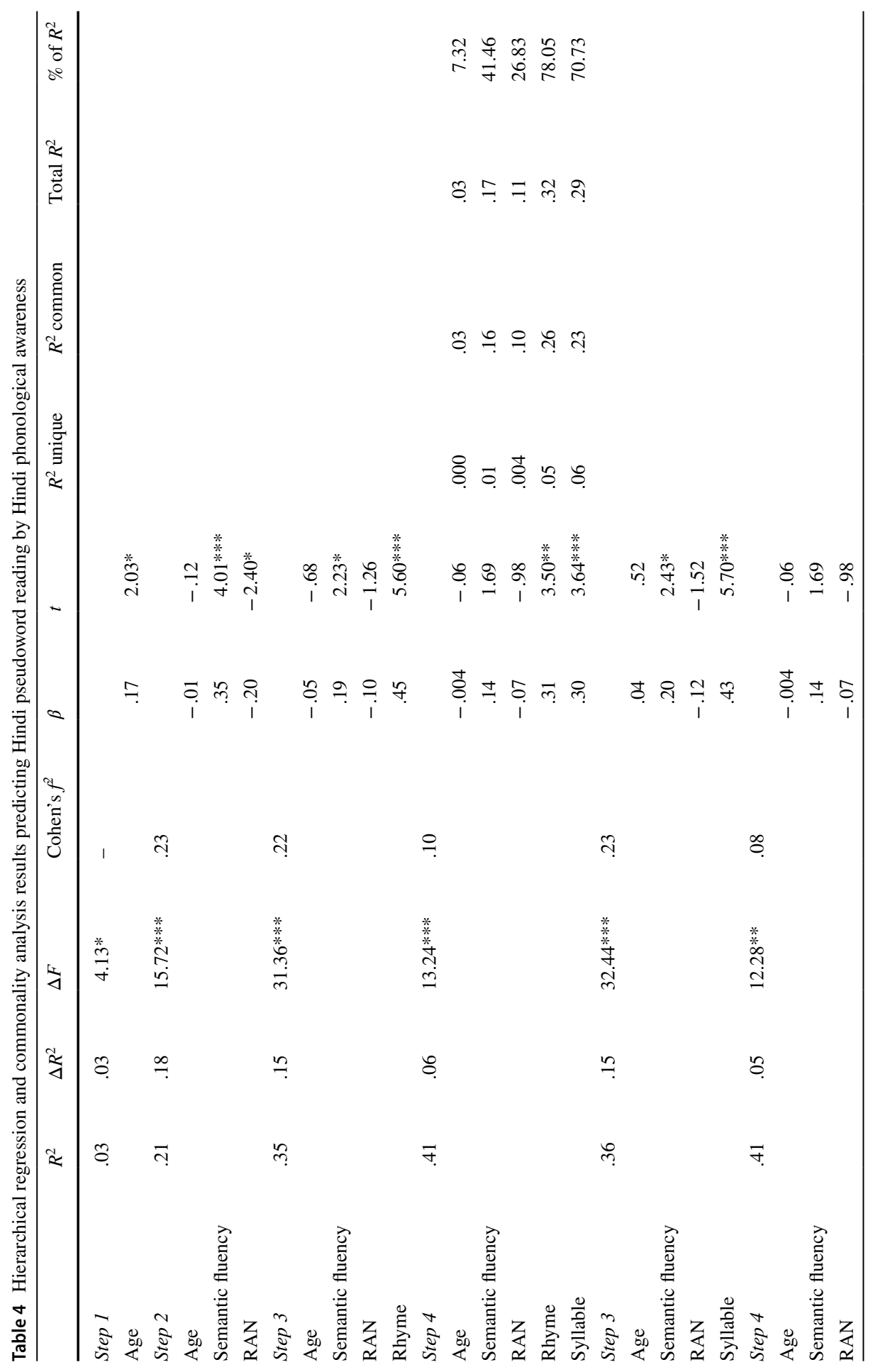




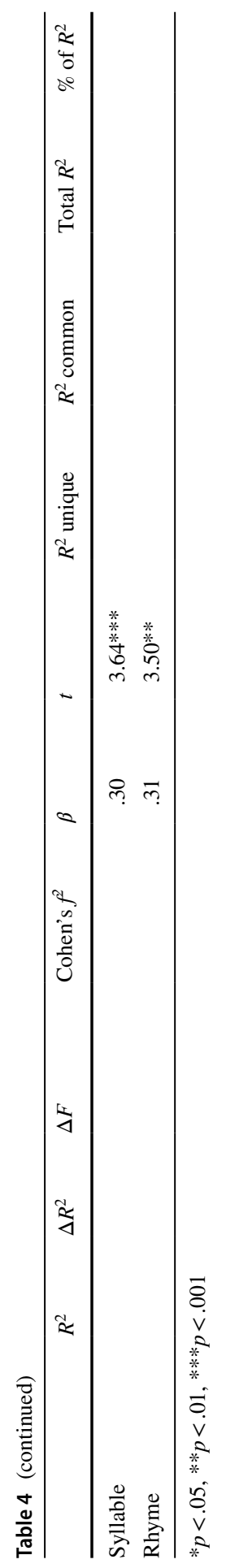

를 Springer 


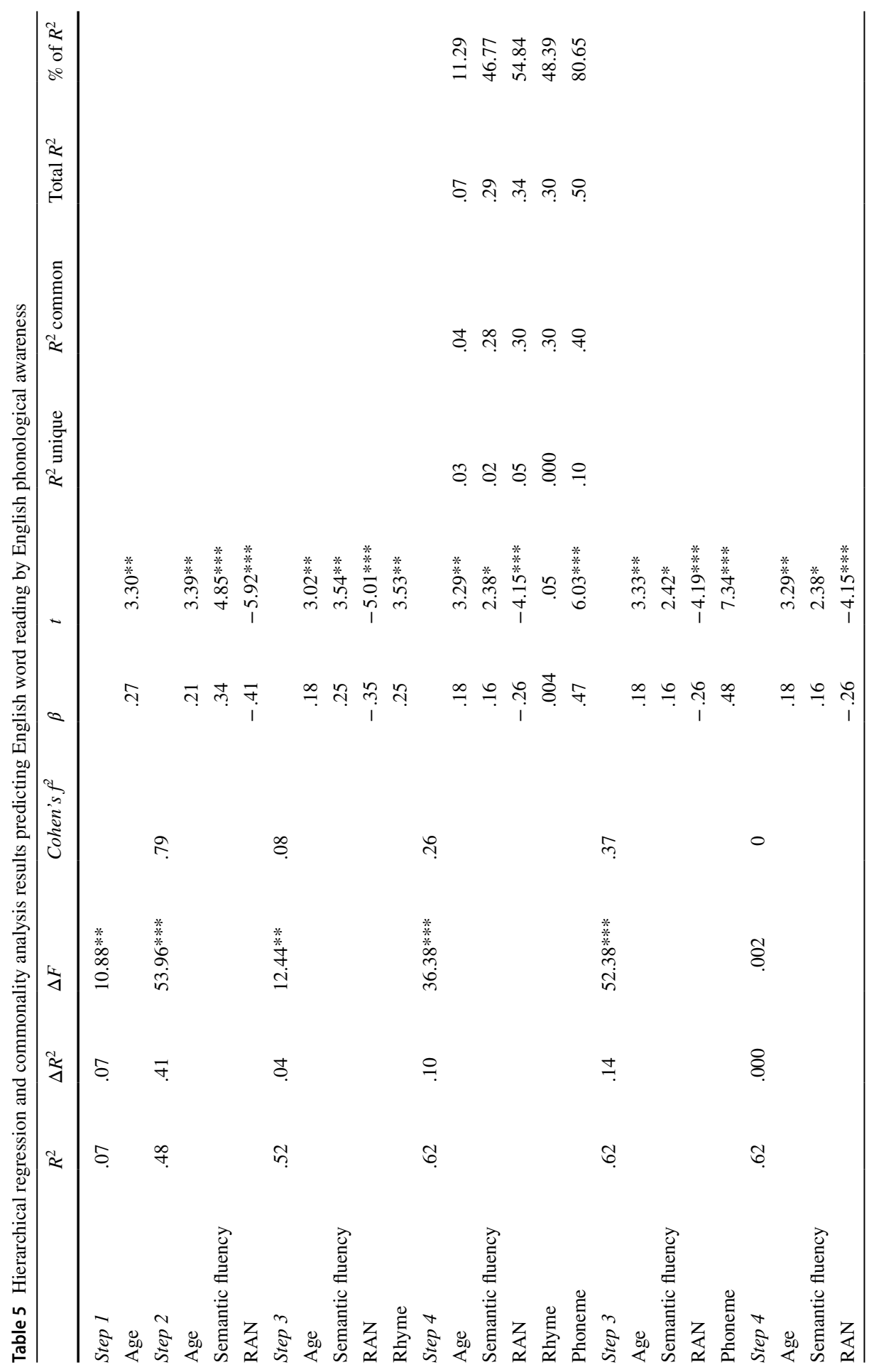




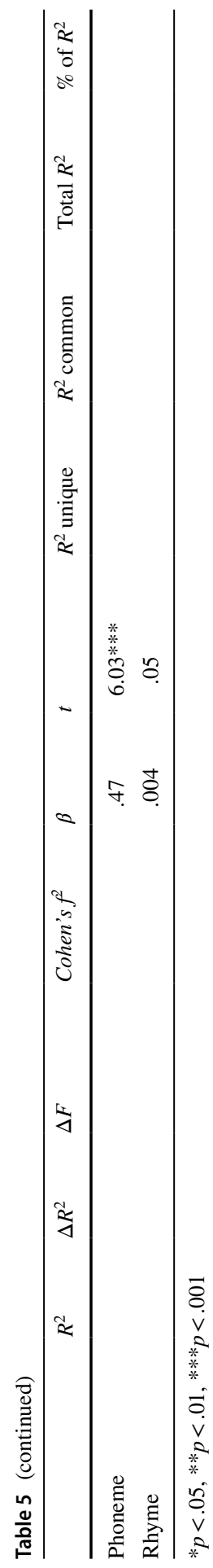

글 Springer 


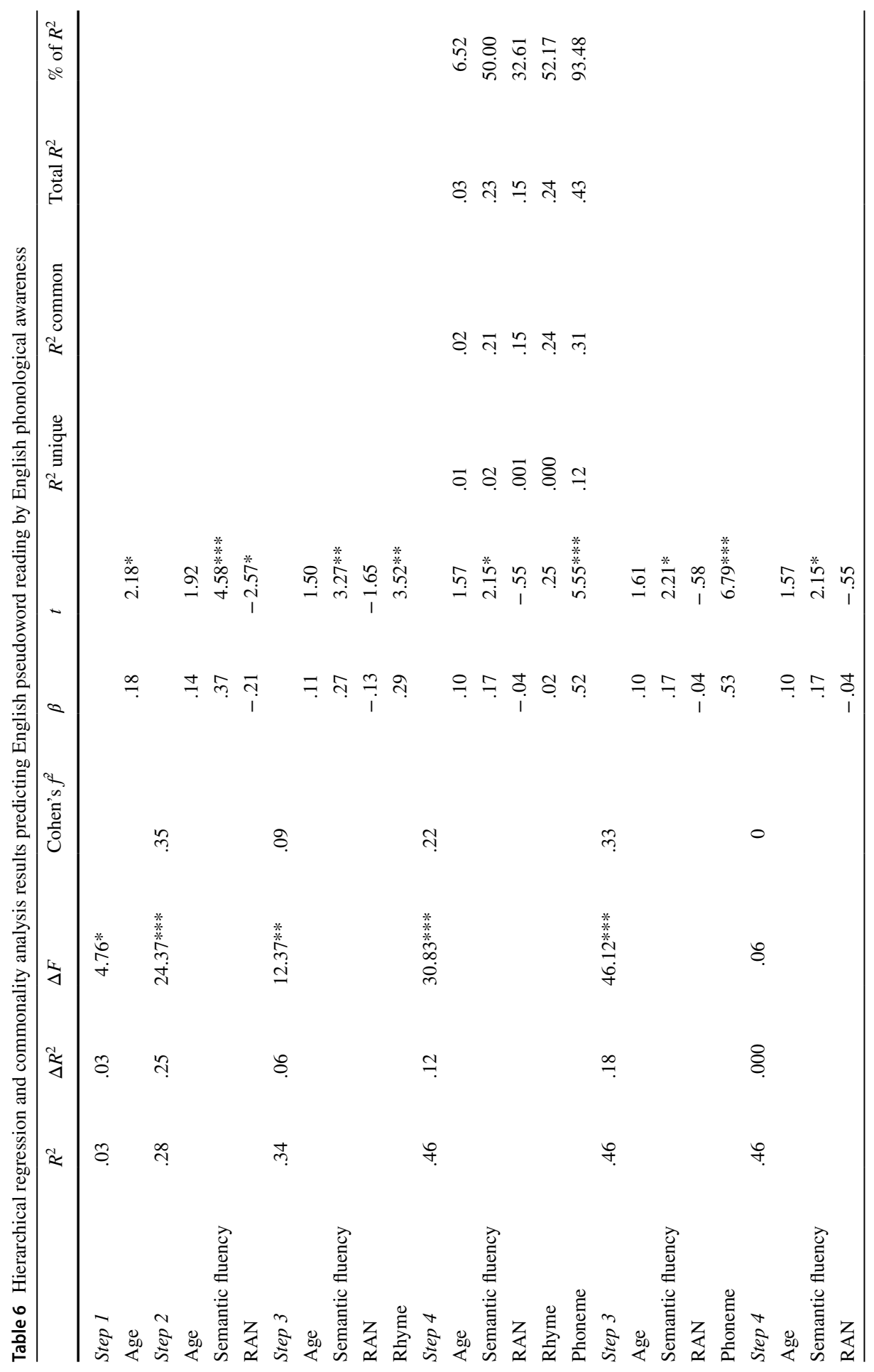




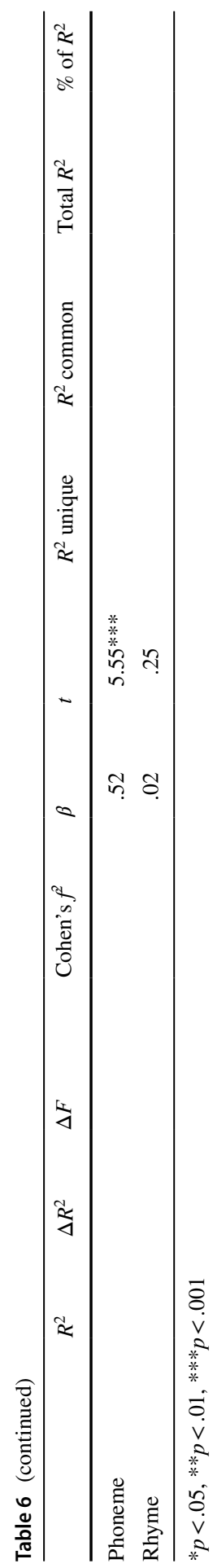

글 Springer 
In predicting Hindi pseudoword reading (see Table 4), the total variance explained by the model was $41 \%$, with the PA measures explaining $20 \%$ of the variance over and above the covariates. Once again, both Hindi PA tasks were uniquely associated with Hindi pseudoword reading. Hindi rhyme oddity uniquely explained 5\% of the variance and accounted for $78 \%$ of the total regression effect. Hindi syllable replacement uniquely explained $6 \%$ of the variance and accounted for $71 \%$ of the total regression effect (Table 4, Step 4).

\section{Predicting English decoding with English PA}

Next, we examined the contribution of the English PA tasks to English word and pseudoword reading (see Tables 5, 6 respectively). Once again, age was entered into the model first, followed by English semantic fluency and English RAN as covariates. The English phonological measures were then entered in alternating order to examine the contribution of each, over and above the other (Steps 3 and 4).

In predicting English word reading (see Table 5), the model explained $62 \%$ of the variance with the PA tasks explaining 14\% of the variance over and above the covariates. When rhyme oddity was the only English PA task entered into the model, it was uniquely associated with English word reading $(\beta=0.25, t=3.53, p<0.001)$. However, once phoneme replacement was entered into the model, rhyme oddity was no longer uniquely associated with English word reading $(\beta=0.004, t=0.05$, $p=0.96)$. English phoneme replacement ultimately emerged as the strongest unique predictor of English word reading $(\beta=0.47, t=6.03, p<0.001)$. Commonality analysis indicated phoneme replacement uniquely explained $10 \%$ of the variance in word reading and accounted for more than $80 \%$ of the regression effect, whereas rhyme oddity has a near-zero unique contribution to the model (Table 5, Step 4).

In predicting English pseudoword reading (see Table 6), the model explained $46 \%$ of the variance, with the PA tasks explaining $18 \%$ of the variance over and above the covariates. Once again, rhyme oddity was uniquely associated with English pseudoword reading when it was the only PA task entered into the model $(\beta=0.29$, $t=3.52, p<0.01)$. However, once phoneme replacement was entered into the model, the unique contribution of the rhyme oddity task was no longer significant $(\beta=0.02$, $t=0.25, p=0.81)$. The English phoneme replacement task emerged as the strongest unique predictor of English pseudoword reading $(\beta=0.52, t=5.55, p<0.001)$. Commonality analysis indicated phoneme replacement uniquely explained $12 \%$ of the variance in pseudoword reading and accounted for $93 \%$ of the regression effect.

\section{Predicting English decoding with Hindi PA}

We then examined the contribution of Hindi (L1) PA to English (L2) word (Table 7) and pseudoword (Table 8) reading. Once again, hierarchical regression analysis was conducted with age, English semantic fluency, and English RAN entered first as covariates. In models 1 and 2, the Hindi phonological measures 
Table 7 Hierarchical regression and commonality analysis results predicting English word reading by Hindi and English phonological awareness

\begin{tabular}{|c|c|c|c|c|c|c|c|c|}
\hline & $R^{2}$ & $\Delta R^{2}$ & $\Delta F$ & Cohen's $f^{2}$ & $R^{2}$ unique & $R^{2}$ common & Total $R^{2}$ & $\%$ of $R^{2}$ \\
\hline \multicolumn{9}{|l|}{ Model 1} \\
\hline 1. Age & .07 & .07 & $10.88^{* *}$ & & .02 & .05 & .07 & 11.86 \\
\hline 2. E Semantic fluency & .48 & .41 & $53.96 * * *$ & .79 & .05 & .24 & .29 & 49.15 \\
\hline E RAN & & & & & .05 & .29 & .34 & 57.63 \\
\hline 3. H Rhyme & .58 & .10 & $32.38 * * *$ & .24 & .05 & .32 & .37 & 62.71 \\
\hline 4. H Syllable & .59 & .02 & $5.39^{*}$ & .02 & .02 & .28 & .29 & 49.15 \\
\hline \multicolumn{9}{|l|}{ Model 2} \\
\hline 3. H Syllable & .54 & .07 & $19.90 * * *$ & .13 & & & & \\
\hline 4. H Rhyme & .59 & .05 & $16.64 * * *$ & .12 & & & & \\
\hline \multicolumn{9}{|l|}{ Model 3} \\
\hline 1. Age & .07 & .07 & $10.88^{* *}$ & & .02 & .05 & .07 & 10.64 \\
\hline 2. E Semantic fluency & .48 & .41 & $53.96 * * *$ & .79 & .02 & .27 & .29 & 45.31 \\
\hline E RAN & .07 & .07 & $10.88^{* *}$ & & .04 & .31 & .34 & 53.13 \\
\hline 3. E Phoneme & .62 & .14 & $52.38 * * *$ & .37 & .05 & .45 & .50 & 78.13 \\
\hline 4. H Rhyme & .63 & .02 & $5.77 *$ & .03 & .01 & .36 & .37 & 57.81 \\
\hline 5. H Syllable & .64 & .004 & 1.41 & .03 & .004 & .29 & .29 & 45.31 \\
\hline \multicolumn{9}{|l|}{ Model 4} \\
\hline 3. E Phoneme & .62 & .14 & $52.38 * * *$ & .37 & & & & \\
\hline 4. H Syllable & .63 & .01 & 3.14 & .03 & & & & \\
\hline 5. H Rhyme & .64 & .01 & $3.98^{*}$ & .03 & & & & \\
\hline
\end{tabular}

$E$ English, $H$ Hindi

$* p<.05, * * p<.01, * * * p<.001$

were entered to examine the contribution of L1 PA to L2 word reading. Both Hindi rhyme oddity and syllable replacement made a significant unique contribution to English word reading, with Hindi rhyme oddity emerging as the strongest predictor of English word reading in both models $(\beta=0.29, t=4.08, p<0.001)$. Commonality analysis indicated that Hindi rhyme uniquely explained $5 \%$ of the variance in English word reading and accounted for $63 \%$ of the total regression effect. Hindi syllable replacement uniquely explained 2\% of the variance in English word reading and accounted for $49 \%$ of the total regression effect.

We then entered the Hindi PA measures after English phoneme awareness, the strongest within-language predictor of English decoding, to examine the contribution of Hindi PA over and above English PA (see Table 7, Models 3 and 4). Hindi rhyme oddity contributed a significant amount of unique variance to English word reading when it was the only Hindi PA task in the model $(\beta=0.17$, $t=2.10, p<0.05)$. Even after Hindi syllable awareness was added, the contribution of Hindi rhyme remained ( $\beta=0.15, t=2.00 ; p<0.05)$. Hindi rhyme uniquely explained $1 \%$ of the variance in English word reading and accounted for 58\% 
Table 8 Hierarchical regression and commonality analysis results predicting English pseudoword reading by Hindi and English phonological awareness

\begin{tabular}{|c|c|c|c|c|c|c|c|c|}
\hline & $R^{2}$ & $\Delta R^{2}$ & $\Delta F$ & Cohen's $f^{2}$ & $R^{2}$ unique & $R^{2}$ common & Total $R^{2}$ & $\%$ of $R^{2}$ \\
\hline \multicolumn{9}{|l|}{ Model 1} \\
\hline 1. Age & .03 & .03 & $4.76^{*}$ & & .01 & .03 & .03 & 7.89 \\
\hline 2. E Semantic fluency & .28 & .25 & $24.37 * * *$ & .35 & .06 & .16 & .23 & 60.53 \\
\hline E RAN & & & & & .005 & .15 & .15 & 39.47 \\
\hline 3. H Rhyme & .36 & .08 & $16.79 * * *$ & .13 & .04 & .20 & .24 & 63.16 \\
\hline 4. H Syllable & .38 & .01 & 3.00 & .03 & .01 & .18 & .19 & 50.00 \\
\hline \multicolumn{9}{|l|}{ Model 2} \\
\hline 3. H Syllable & .34 & .05 & $11.15^{* *}$ & .09 & & & & \\
\hline 4. H Rhyme & .38 & .04 & $8.29 * *$ & .06 & & & & \\
\hline \multicolumn{9}{|l|}{ Model 3} \\
\hline 1. Age & .03 & .03 & $4.76^{*}$ & & .01 & .03 & .03 & 6.38 \\
\hline 2. E Semantic fluency & .28 & .25 & $24.37 * * *$ & .35 & .02 & .21 & .23 & 48.94 \\
\hline E RAN & & & & & .001 & .15 & .15 & 31.91 \\
\hline 3. E Phoneme & .46 & .18 & $46.17 * * *$ & .33 & .09 & .34 & .43 & 91.49 \\
\hline 4. H Rhyme & .47 & .002 & .63 & .02 & .002 & .23 & .24 & 51.06 \\
\hline 5. H Syllable & .47 & .001 & .20 & 0 & .001 & .19 & .19 & 40.43 \\
\hline \multicolumn{9}{|l|}{ Model 4} \\
\hline 3. E Phoneme & .46 & .18 & $46.17 * * *$ & .33 & & & & \\
\hline 4. H Syllable & .47 & .002 & .42 & .02 & & & & \\
\hline 5. H Rhyme & .47 & .002 & .41 & 0 & & & & \\
\hline
\end{tabular}

$E$ English, $H$ Hindi

$* p<.05, * * p<.01, * * * p<.001$

of the total regression effect. Hindi syllable replacement contributed near zero unique variance to English word reading.

In predicting English pseudoword reading (see Table 8), Hindi rhyme oddity contributed significant unique variance in both models 1 and $2(\beta=0.25, t=2.88$, $p<0.01)$. On the contrary Hindi syllable awareness added significant unique variance when entered before Hindi rhyme (see Table 8, Model 2) but not when entered after Hindi rhyme (see Table 8, Model 1). Commonality analysis indicated that Hindi rhyme uniquely explained $4 \%$ of the variance in English pseudoword reading and accounted for $63 \%$ of the total regression effect. Hindi syllable replacement, while only adding a small amount of unique variance (1\%), accounted for $50 \%$ of the total regression effect. Neither Hindi rhyme nor Hindi syllable awareness added any significant unique variance to English pseudoword reading when English phoneme awareness was included in the model. 
Table 9 Hierarchical regression and commonality analysis results predicting Hindi word reading by Hindi and English phonological awareness

\begin{tabular}{|c|c|c|c|c|c|c|c|c|}
\hline & $R^{2}$ & $\Delta R^{2}$ & $\Delta F$ & Cohen's $f^{2}$ & $R^{2}$ Unique & $R^{2}$ Common & Total $R^{2}$ & $\%$ of $R^{2}$ \\
\hline \multicolumn{9}{|l|}{ Model 1} \\
\hline 1. Age & .06 & .06 & $9.59 * *$ & & .01 & .06 & .06 & 16.22 \\
\hline 2. H Semantic fluency & .25 & .19 & $17.49 * * *$ & .25 & .02 & .16 & .18 & 48.65 \\
\hline H RAN & & & & & .03 & .13 & .16 & 43.24 \\
\hline 3. E Rhyme & .30 & .05 & $9.16^{* *}$ & .07 & .000 & .18 & .18 & 48.65 \\
\hline 4. E Phoneme & .37 & .08 & $16.62 * * *$ & .11 & .08 & .20 & .28 & 75.68 \\
\hline \multicolumn{9}{|l|}{ Model 2} \\
\hline 3. E Phoneme & .37 & .12 & $27.00 * * *$ & .19 & & & & \\
\hline 4. E Rhyme & .37 & .000 & .001 & 0 & & & & \\
\hline \multicolumn{9}{|l|}{ Model 3} \\
\hline 1. Age & .06 & .06 & $9.59 * *$ & & .01 & .06 & .06 & 13.95 \\
\hline 2. H Semantic fluency & .25 & .19 & $17.49 * * *$ & .25 & .01 & .17 & .18 & 41.86 \\
\hline H RAN & & & & & .02 & .14 & .16 & 37.21 \\
\hline 3. H Rhyme & .36 & .11 & $23.36^{* * * *}$ & .17 & .01 & .28 & .29 & 67.44 \\
\hline 4. E Phoneme & .40 & .04 & $8.86^{* *}$ & .07 & .01 & .26 & .28 & 65.12 \\
\hline 5. H Syllable & .43 & .03 & $8.08 * *$ & .05 & .03 & .24 & .28 & 65.12 \\
\hline \multicolumn{9}{|l|}{ Model 4} \\
\hline 3. H Syllable & .39 & .13 & $30.06^{* * *}$ & .23 & & & & \\
\hline 4. E Phoneme & .42 & .04 & $8.28 * *$ & .05 & & & & \\
\hline 5. H Rhyme & .43 & .01 & 2.89 & .02 & & & & \\
\hline \multicolumn{9}{|l|}{ Model 5} \\
\hline 3. H Rhyme & .36 & .11 & $23.36^{* * * *}$ & .17 & & & & \\
\hline 4. H Syllable & .42 & .06 & $13.69 * * *$ & .10 & & & & \\
\hline 5. E Phoneme & .43 & .01 & 3.46 & .02 & & & & \\
\hline
\end{tabular}

$E$ English, $H$ Hindi

$* p<.05, * * p<.01, * * * p<.001$

\section{Predicting Hindi decoding with English PA}

Finally, we examined the contribution of English (L2) PA to Hindi (L1) word (Table 9) and pseudoword (Table 10) reading. Hierarchical regression analysis was conducted, this time with age, Hindi semantic fluency, and Hindi RAN entered as covariates. Next, the English phonological measures were entered in alternating order to examine the contribution of L2 PA to L1 word reading (see Table 9, Models 1 and 2). English rhyme oddity added significant unique variance when entered before English phoneme replacement (Table 9, Model 1) but not when entered after English phoneme replacement (Table 9, Model 2). Commonality analysis indicated a near-zero unique contribution of English rhyme oddity. English phoneme replacement added significant unique variance in both models $(\beta=0.39, t=4.08, p<0.001)$, 
Table 10 Hierarchical regression and commonality analysis results predicting Hindi pseudoword reading by Hindi and English phonological awareness

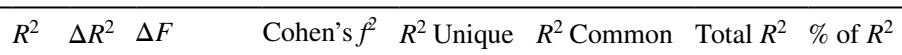

\begin{tabular}{|c|c|c|c|c|c|c|c|c|}
\hline 1. Age & .03 & .03 & $4.13^{*}$ & & .000 & .03 & .03 & 7.89 \\
\hline 2. H Semantic fluency & .21 & .18 & $15.72 * * *$ & .23 & .02 & .15 & .17 & 44.74 \\
\hline H RAN & & & & & .01 & .10 & .11 & 28.95 \\
\hline 3. E Rhyme & .31 & .10 & $19.46 * * *$ & .14 & .01 & .23 & .24 & 63.16 \\
\hline 4. E Phoneme & .38 & .08 & $16.99 * * *$ & .11 & .08 & .24 & .32 & 84.21 \\
\hline \multicolumn{9}{|l|}{ Model 2} \\
\hline 3. E Phoneme & .38 & .17 & $37.23 * * *$ & .27 & & & & \\
\hline 4. E Rhyme & .38 & .01 & 1.37 & 0 & & & & \\
\hline \multicolumn{9}{|l|}{ Model 3} \\
\hline 1. Age & .03 & .03 & $4.13 *$ & & .000 & .03 & .03 & 6.82 \\
\hline 2. H Semantic fluency & .21 & .18 & $15.72 * * *$ & .23 & .01 & .16 & .17 & 38.64 \\
\hline H RAN & & & & & .003 & .10 & .11 & 25.00 \\
\hline 3. H Rhyme & .35 & .15 & $31.36^{* * * *}$ & .22 & .02 & .30 & .32 & 72.73 \\
\hline 4. E Phoneme & .41 & .05 & $12.59 * * *$ & .10 & .03 & .30 & .32 & 72.73 \\
\hline 5. H Syllable & .44 & .03 & $6.76^{* *}$ & .05 & .03 & .26 & .29 & 65.91 \\
\hline \multicolumn{9}{|l|}{ Model 4} \\
\hline 3. H Syllable & .36 & .15 & $32.44 * * *$ & .23 & & & & \\
\hline 4. E Phoneme & .42 & .06 & $14.03 * * *$ & .10 & & & & \\
\hline 5. H Rhyme & .44 & .02 & $4.50^{*}$ & .04 & & & & \\
\hline \multicolumn{9}{|l|}{ Model 5} \\
\hline 3. H Rhyme & .35 & .15 & $31.36 * * *$ & .22 & & & & \\
\hline 4. H Syllable & .41 & .06 & $13.24 * * *$ & .10 & & & & \\
\hline 5. E Phoneme & .44 & .03 & 6.15 & .05 & & & & \\
\hline
\end{tabular}

$E$ English, $H$ Hindi

$* p<.05, * * p<.01, * * * p<.001$

uniquely explaining $8 \%$ of the variance in Hindi reading and accounting for over $75 \%$ of the total regression effect.

We then entered English phoneme replacement after the two Hindi PA measures to examine the contribution of English phoneme awareness over and above Hindi PA (see Table 9, Models 3, 4, and 5). English phoneme replacement added a significant amount of unique variance to Hindi word reading when entered after Hindi rhyme (Table 9, Model 3) and when entered after Hindi syllable replacement (Table 9, Model 4), but did not add any significant unique variance when added after both Hindi PA tasks (Table 9, Model 5). Commonality analysis indicated that English PA uniquely explains $1 \%$ of the variance in Hindi word reading.

In predicting Hindi pseudoword reading (see Table 10), once again English phoneme awareness contributed significant unique variance in both models 1 and $2(\beta=0.38, t=4.12, p<0.001)$. On the contrary, English rhyme oddity only 
added significant unique variance when added before English phoneme awareness (Table 10, Model 1). Commonality analysis indicated that English phoneme replacement uniquely explained $8 \%$ of the variance in Hindi pseudoword reading and accounted for $84 \%$ of the regression effect. English rhyme oddity also added a small amount of unique variance to the model.

We then entered English phoneme replacement after the two Hindi PA measures to examine the contribution of English phoneme awareness over and above Hindi PA (see Table 10, Models 3, 4, and 5). English phoneme replacement added a significant amount of unique variance to Hindi pseudoword reading when entered after Hindi rhyme (Table 10, Model 3) and when entered after Hindi syllable replacement (Table 10, Model 4), but did not add any significant unique variance when added after both Hindi PA tasks (Table 10, Model 5). Commonality analysis indicated that English PA uniquely explained 3\% of the variance in Hindi pseudoword reading.

\section{Discussion}

In this study we aimed to examine cross-language transfer of PA among emergent readers in India. Children in Grades 1 and 2 were simultaneously exposed to Hindi and English literacy instruction in school and were expected to learn to read in two distinct writing systems. Previous studies on cross-language transfer in India which have focused on children in Grade 4 and above have found evidence of L1 phonological transfer to L2 reading (Reddy \& Koda, 2013), as well as vice versa (Mishra \& Stainthorp, 2007). The results of our study extend these findings to include emergent readers in Grades 1 and 2.

In both Hindi and English, the PA measures of a language were predictive of decoding in that language, highlighting the importance of PA in reading across languages (e.g., Goswami, 2008). Both Hindi PA tasks added a significant amount of unique variance to Hindi word and pseudoword reading, a finding in line with previous studies which have indicated a critical role of PA, particularly large-unit awareness in akshara decoding (Nag, 2007; Nag \& Snowling, 2012; Nakamura et al., 2018; Singh \& Sumathi, 2019). As previously mentioned, although information is encoded at both the syllable and phoneme-levels in akshara orthographies, it is the syllable that is the salient unit. Early literacy instruction in the akshara languages also tends to focus on syllable-level mappings (Nag, 2007, 2011). As a result, it is perhaps unsurprising that the Hindi syllable replacement task emerged as the strongest predictor of Hindi word reading. Pseudoword reading in Hindi was highly correlated with Hindi word reading, reflective of the orthographic transparency present in akshara languages (Singh \& Sumathi, 2019). Consequently, both Hindi phonological tasks were also significant unique predictors of pseudoword reading in Hindi.

When predicting English decoding, English phoneme-level awareness emerged as the single strongest predictor of English decoding. English rhyme oddity, on the other hand, only significantly contributed to English decoding when it was the only PA measure in the model but added no additional variance after controlling for the effect of phonemic awareness. This finding is in line with previous 
research examining Kannada-English biliteracy among older children (Reddy \& Koda, 2013). Ample studies have identified phonemic awareness as one of the most important predictors of reading in alphabetic languages such as English (e.g., Melby-Lervåg et al., 2012), and many studies have shown the benefits of phonemic awareness instruction in helping children read in English (Ehri et al., 2001). The findings of our study show that even for young English language learners with an akshara-based L1, English phoneme awareness is critical for English decoding. Thus, the role of English phoneme awareness is one that cannot, and should not, be ignored in English-medium classrooms in India.

Both Hindi PA measures significantly predicted English decoding when English phoneme awareness was not included in the model, a finding once again in line with previous work examining older students in India (e.g., Reddy \& Koda, 2013). When English phoneme awareness was entered into the model, Hindi rhyme oddity continued to add a small but significant amount of unique variance to English word reading. These findings extend previous studies on aksharaEnglish biliteracy and are in line with studies in other language pairs (e.g. Chinese-English; Chow et al., 2005) to show that even in these emergent readers, PA in the L1 transfers to L2 reading. Furthermore, although English decoding is heavily reliant on awareness of the smallest unit, or that of the phoneme, syllablelevel awareness is also important given that English is a highly irregular orthography (Ziegler \& Goswami, 2005). Syllable awareness has long been known to be one of the earliest available phonological units, emerging even before children begin formal literacy instruction (Treiman \& Zukowski, 1991), and is seen as a universal in language processing (Ziegler \& Goswami, 2005). Transfer of these non-language specific aspects of PA is also in line with the Transfer Facilitation Model (Koda, 2008).

Although only the Hindi rhyme task was found to add additional variance, it is important to note that, due to the orthographic characteristics of Hindi, rhyme awareness and syllable awareness are essentially testing awareness at the same grain size, that of the syllable (see Cherodath et al., 2017). This is contrary to the English rhyme oddity task which requires onset-rime sensitivity which is considered to be a finer level of sensitivity (Goswami \& Bryant, 1990; Treiman \& Zukowski, 1991). However, while both Hindi tasks are testing awareness at the level of the syllable, the rhyme task is an identification task whereas the syllable replacement task is a manipulation task. Identification tasks have been recognized as being easier than manipulation tasks (Pufpaff, 2009), a finding that also holds true in this sample where children performed significantly better on the rhyme identification tasks than the manipulation tasks. The contribution of Hindi rhyme, and not syllable replacement, to English word reading may be reflective of differences in task difficulty rather than differences in the type of PA that is being transferred. However, further studies in which the distinction between Hindi syllable and Hindi sub-syllabic units is established are needed to better understand the role of Hindi syllable versus Hindi rhyme awareness and their differential contributions to English decoding.

We also found that phoneme awareness in English (L2) added a significant amount of variance to L1 decoding when L1 PA was not included in the model. However, when L1 PA was added in, there was no longer evidence of transfer from 
L2 PA to L1 decoding. However, commonality analysis indicated this may be due to multicollinearity such that once both L2 and L1 PA are in the model, they both lose predictive power. This finding is in line with previous studies which have identified PA as a language general skill (e.g. Cisero \& Royer, 1995; Comeau et al., 1999; Kim, 2009). Mishra and Stainthorp (2007) found no evidence of L2 to L1 transfer in children who were going to English-medium school. Interestingly however, evidence of L2 to L1 transfer was found among children who were attending Oriya-medium school. Chung et al. (2013) who examined Chinese-English bilinguals similarly found a lack of L2-L1 transfer. They concluded that a lack of transfer may be due to limited L2 proficiency and that perhaps as children's L2 proficiency increases, there may be stronger bidirectional transfer. Studies which have found evidence of L2-L1 transfer have been in line with this conclusion (e.g. LaFrance \& Gottardo, 2005). Future studies are needed to untangle the effects of language proficiency as well as language of instruction in multilingual environments like India.

\section{Limitations}

There are some limitations to the present study which should be considered when interpreting the findings. First, although we attempted to control for oral language skill through the semantic fluency measure, children's oral vocabulary was not explicitly assessed. Oral vocabulary is known to play a critical role in children's PA development and word reading skill (e.g., Metsala \& Walley, 1998), a finding which also holds true for reading in Hindi (Singh \& Sumathi, 2019). In future studies, it is important to assess oral vocabulary in both languages. In addition, using equivalent measures which assess PA at multiple grain sizes (i.e. syllable, onset-rime, and phoneme-levels) in both languages will help provide more specific information on the role of cross-language transfer. Second, the cross-sectional nature of this study prevents us from understanding how associations may change as children develop over time. There is only one longitudinal study, to our knowledge, which has investigated akshara-English biliteracy development in an Indian sample (see Nakamura et al., 2014). However, the youngest group of children in this study were in Grade 3. Thus, future studies are needed in which children's literacy development is followed starting at school entry. Third, this study only reports correlational data. Future studies which employ randomized controlled trials to study the effects of PA and reading instruction can provide valuable insights on how to improve reading development. Finally, it is important to acknowledge the characteristics of the sample when interpreting the generalizability of the results. India is a highly diverse country with an education system that differs greatly from school to school and state to state. Future research should examine cross-language associations in a wide variety of age groups, language pairs, and school types across the country to build a stronger evidence base which is applicable to the Indian population. 


\section{Implications}

Despite these limitations, this study does provide meaningful insight into the crosslanguage relationships of young Hindi-English biliterates, a population that has been greatly understudied. While we would encourage researchers to examine this population in greater detail there are important implications, both theoretical and practical, that have emerged from this study. Theoretically, we have shown that L1 and L2 PA transfers to L1 and L2 decoding even among emergent akshara-English bilinguals. This study expands upon previous research on cross-language transfer among akshara-English bilinguals which has focused on children in Grade 4 and above, once children have well developed literacy skills. Through the examination of a younger set of students (Grade 1 and 2) who were simultaneously learning to read in both their L1 and L2 we were able to highlight the importance of PA for decoding both within and across languages. These findings have important practical implications for effective literacy instruction in India.

We see that the role that English phonemic awareness plays in children's English decoding skills, even in this population, is one that cannot be ignored. In line with previous work, it is clear that explicit and systematic instruction that supports the development of English phonemic awareness can successfully aid English reading development (e.g., Dixon et al., 2011). We would encourage teachers and practitioners to better leverage children's L1 literacy skills in helping them develop their L2 literacy skills. As mentioned by Reddy and Koda (2013), this could involve explicitly highlighting the sub-lexical components which are used in both languages. In a study conducted with 10-year-old Kannada-speaking children, researchers found that when an English phonics intervention was modified to include the Kannada symbols which represent the English letters, children performed significantly better on English reading as compared to children who received the English-only intervention (Nishanimut et al., 2013). These findings are promising, although future studies are needed in which such interventions are tested with emergent readers.

We would like to conclude by acknowledging those who have provided sound arguments against the introduction of English prior to the sufficient development of children's literacy skills in their L1 (Nakamura et al., 2019). Studies have shown the role of transfer to be much more significant once children achieve a "threshold level" of literacy in their L1, and thus, researchers are encouraging practitioners and policy makers alike to not introduce English decoding instruction until children have reached the threshold level. Paying heed to these findings, India's New National Education policy has recommended that the medium of instruction until at least Grade 5, but preferably until Grade 8, be the mother tongue or the local language (Government of India Ministry of Education, 2020). However, this remains a recommendation leaving state governments free to decide when and where it will be implemented (Vishnoi, 2020). In India, English-medium instruction starting at school entry is clearly there to stay. Therefore, it is critical that researchers continue to examine literacy development among these populations. 
Acknowledgements We would like to thank the school, principal, teachers, parents, and students for their cooperation and participation in this study. We would also like to extend a heartfelt thanks to our research assistants (Ms. Mahima Bhalla, Ms. Samriddhi Dhiman, \& Ms. Saumya Goel) for their support during data collection and Ms. Sandhya Saxena (UNESCO MGIEP) for her support in the translation of various documents used in this study.

Authors' contributions Data for this study was collected by Priyanka Patel as part of her doctoral dissertation. Minna Torppa is Priyanka Patel's doctoral supervisor and supported the planning of data collection, data analysis and the writing of the paper. Nandini Chatterjee Singh provided her expertise on Hindi literacy and supported the writing and proofreading of the paper. She also provided all the measures which were used for data collection.

Funding Open Access funding provided by University of Jyväskylä (JYU). Funding for this study was provided by the University of Jyväskylä.

Availability of data and materials Data and material can be provided by the authors upon request.

Code availability Not applicable.

\section{Declarations}

Conflict of interest The authors declare that the research has been conducted in the absence of any relationships that could be construed as a potential conflict of interest.

Ethical approval The study was carried out in accordance with the guidelines provided by the University of Jyväskylä Ethics Committee. A formal ethics approval was not required as per the University of Jyväskylä Ethics Committee guidelines and national regulations, but a statement from the ethics committee can be provided upon request.

Consent to participate Written informed consent was collected from the parents for their child's participation in the study. In addition, permission to conduct the data collection was also taken from the school principal and the respective class teachers.

Consent for publication Not applicable.

Open Access This article is licensed under a Creative Commons Attribution 4.0 International License, which permits use, sharing, adaptation, distribution and reproduction in any medium or format, as long as you give appropriate credit to the original author(s) and the source, provide a link to the Creative Commons licence, and indicate if changes were made. The images or other third party material in this article are included in the article's Creative Commons licence, unless indicated otherwise in a credit line to the material. If material is not included in the article's Creative Commons licence and your intended use is not permitted by statutory regulation or exceeds the permitted use, you will need to obtain permission directly from the copyright holder. To view a copy of this licence, visit http://creativecommons.org/licen ses/by/4.0/.

\section{References}

Adams, M. (1990). Beginning to read: Thinking and learning about print. MIT Press.

Anthony, J. L., \& Lonigan, C. J. (2004). The nature of phonological awareness: Converging evidence from four studies of preschool and early grade school children. Journal of Educational Psychology, 96(1), 43-55. https://doi.org/10.1037/0022-0663.96.1.43 
Branum-Martin, L., Tao, S., \& Garnaat, S. (2015). Bilingual phonological awareness: Reexamining the evidence for relations within and across languages. Journal of Educational Psychology, 107(1), 111-125. https://doi.org/10.1037/a0037149

Branum-Martin, L., Tao, S., Garnaat, S., Bunta, F., \& Francis, D. J. (2012). Meta-analysis of bilingual phonological awareness: Language, age, and psycholinguistic grain size. Journal of Educational Psychology, 104(4), 932-944. https://doi.org/10.1037/a0027755

Bruck, M., \& Genesee, F. (1995). Phonological awareness in young second language learners. Journal of Child Language, 22(2), 307-324. https://doi.org/10.1017/S0305000900009806

Carroll, J. M. (2001). The development of phonological awareness in pre-school children. [Doctoral dissertation, University of York]. https://etheses.whiterose.ac.uk/9761/1/251812.pdf

Chen, X., Xu, F., Nguyen, T.-K., Hong, G., \& Wang, Y. (2010). Effects of cross-language transfer on first-language phonological awareness and literacy skills in Chinese children receiving English instruction. Journal of Educational Psychology, 102(3), 712-728. https://doi.org/10.1037/a0018802

Cherodath, S., Rao, C., Midha, R., Sumathi, T. A., \& Singh, N. (2017). A role for putamen in phonological processing in children. Bilingualism: Language and Cognition, 20(2), 318-326. https://doi.org/ $10.1017 /$ S1366728916000614

Cherodath, S., \& Singh, N. C. (2015). The influence of orthographic depth on reading networks in simultaneous biliterate children. Brain and Language, 143, 42-51. https://doi.org/10.1016/j.bandl.2015. 02.001

Chow, B.W.-Y., McBride-Chang, C., \& Burgess, S. (2005). Phonological processing skills and early reading abilities in Hong Kong Chinese kindergarteners learning to read English as a second language. Journal of Educational Psychology, 97(1), 81-87. https://doi.org/10.1037/0022-0663.97.1.81

Chung, K. K., McBride-Chang, C., Cheung, H., \& Wong, S. W. (2013). General auditory processing, speech perception and phonological awareness skills in Chinese-English biliteracy. Journal of Research in Reading, 36(2), 202-222. https://doi.org/10.1111/j.1467-9817.2011.01500.x

Cisero, C. A., \& Royer, J. M. (1995). The development and cross-language transfer of phonological awareness. Contemporary Educational Psychology, 20(3), 275-303. https://doi.org/10.1006/ceps. 1995.1018

Cohen, J. (1992). A power primer. Psychological Bulletin, 112, 155-159. https://doi.org/10.1037/00332909.112.1.155

Comeau, L., Cormier, P., Grandmaison, É., \& Lacroix, D. (1999). A longitudinal study of phonological processing skills in children learning to read in a second language. Journal of Educational Psychology, 91(1), 29. https://doi.org/10.1037/0022-0663.91.1.29

Compton, D. L. (2003). Modeling the relationship between growth in rapid naming speed and growth in decoding skill in first-grade children. Journal of Educational Psychology, 95(2), 225-239. https:// doi.org/10.1037/0022-0663.95.2.225

Cooper, D. H., Roth, F. P., Speece, D. L., \& Schatschneider, C. (2002). The contribution of oral language skills to the development of phonological awareness. Applied Psycholinguistics, 23(3), 399-416. https://doi.org/10.1017/S0142716402003053

Dixon, P., Schagen, I., \& Seedhouse, P. (2011). The impact of an intervention on children's reading and spelling ability in low-income schools in India. School Effectiveness and School Improvement, 22(4), 461-482. https://doi.org/10.1080/09243453.2011.625125

Dongre, A., \& Tewary, V. (2014). Impact of private tutoring on learning levels: Evidence from India. Accountability Initiative. http://ideasforindia.in/article.aspx?article_id=319\#sthash.TffWe6Zs. dpuf/

Durgunoğlu, A. Y., Nagy, W. E., \& Hancin-Bhatt, B. J. (1993). Cross-language transfer of phonological awareness. Journal of Educational Psychology, 85(3), 453-465. https://doi.org/10.1037/ $0022-0663.85 .3 .453$

Dutta, U., \& Bala, N. (2012). Teaching of English at primary level in government schools. NCERT. http://www.ncert.nic.in/departments/nie/del/publication/pdf/English_Primary_level.pdf

Ehri, L. C., Nunes, S. R., Stahl, S. A., \& Willows, D. M. (2001). Systematic phonics instruction helps students learn to read: Evidence from the National Reading Panel's meta-analysis. Review of Educational Research, 71(3), 393-447. https://doi.org/10.3102/00346543071003393

Frederickson, N., Frith, U., \& Reason, R. (1997). Phonological assessment battery. NFER-Nelson.

Goswami, U. (2008). The development of reading across languages. Annals of the New York Academy of Sciences, 1145, 1-12. https://doi.org/10.1196/annals.1416.018 
Goswami, U. (2010). A psycholinguistic grain size view of reading development across languages. In N. Brunswick, S. McDougall, \& P. de Mornay Davies (Eds.), Reading and dyslexia in different orthographies (pp. 23-42). Psychology Press.

Goswami, U., \& Bryant, P. E. (1990). Phonological skills and learning to read. Lawrence Erlbaum.

Gottardo, A., Chen, X., \& Huo, M. R. Y. (2021). Understanding within-and cross-language relations among language, preliteracy skills, and word reading in bilingual learners: Evidence from the science of reading. Reading Research Quarterly, 56, S371-S390. https://doi.org/10.1002/rrq.410

Gottardo, A., Yan, B., Siegel, L. S., \& Wade-Woolley, L. (2001). Factors related to English reading performance in children with Chinese as a first language: More evidence of cross-language transfer of phonological processing. Journal of Educational Psychology, 93(3), 530-542. https://doi. org/10.1037/0022-0663.93.3.530

Government of India Ministry of Education. (2020). National Education Policy 2020. https://www. education.gov.in/sites/upload_files/mhrd/files/NEP_Final_English_0.pdf

Hatcher, P. J., Hulme, C., \& Ellis, A. W. (1994). Ameliorating early reading failure by integrating the teaching of reading and phonological skills: The phonological linkage hypothesis. Child Development, 65, 41-57. https://doi.org/10.1111/j.1467-8624.1994.tb00733.x

Joshi, R. M., Nakamura, P. R., \& Singh, N. C. (2017). Reading research and practice: Indianperspective. New Dir Child Adolesc Dev, 158, 43-53. https://doi.org/10.1002/cad.20222

Kandhadai, P., \& Sproat, R. (2010). Impact of spatial ordering of graphemes in alphasyllabic scripts on phonemic awareness in Indic languages. Writing Systems Research, 2, 105-116. https://doi. org/10.1093/wsr/wsq009

Kavé, G. (2006). The development of naming and word fluency: Evidence from Hebrew-speaking children between ages 8 and 17. Developmental Neuropsychology, 29(3), 493-508. https://doi. org/10.1207/s15326942dn2903_7

Kim, Y.-S. (2009). Crosslinguistic influence on phonological awareness for Korean-English bilingual children. Reading and Writing, 22(7), 843-861. https://doi.org/10.1007/s11145-008-9132-z

Koda, K. (2007). Cross-linguistic constraints on second-language reading development. In K. Koda (Ed.), Reading and language learning (pp. 1-44). Blackwell Publishing.

Koda, K. (2008). Impacts of prior literacy experience on second language learning to read. In K. Koda \& A. M. Zehler (Eds.), Learning to read across languages: Cross-linguistic relationships in firstand second language literacy development (pp. 68-96). Routledge.

Kraha, A., Turner, H., Nimon, K., Zientek, L., \& Henson, R. (2012). Tools to support interpreting multiple regression in the face of multicollinearity. Frontiers in Psychology, 3, 44. https://doi. org/10.3389/fpsyg.2012.00044

Kuo, L. J., Uchikoshi, Y., Kim, T. J., \& Yang, X. (2016). Bilingualism and phonological wareness: Re-examining theories of cross-language transfer and structural sensitivity. Contemporary Educational Psychology, 46, 1-9. https://doi.org/10.1016/j.cedpsych.2016.03.002

LaFrance, A., \& Gottardo, A. (2005). A longitudinal study of phonological processing skills and reading in bilingual children. Applied Psycholinguistics, 26, 559-578. https://doi.org/10.1017/S0142 716405050307

McBride-Chang, C., Tong, X., Shu, H., Wong, A. M. Y., Leung, K. W., \& Tardif, T. (2008). Syllable, phoneme, and tone: Psycholinguistic units in early Chinese and English word recognition. Scientific Studies of Reading, 12(2), 171-194. https://doi.org/10.1080/10888430801917290

Melby-Lervåg, M., \& Lervåg, A. (2011). Cross-linguistic transfer of oral language, decoding, phonological awareness and reading comprehension: A meta-analysis of the correlational evidence. Journal of Research in Reading, 34(1), 114-135. https://doi.org/10.1111/j.1467-9817.2010.01477.x

Melby-Lervåg, M., Lyster, S. A. H., \& Hulme, C. (2012). Phonological skills and their role in learning to read: A meta-analytic review. Psychological Bulletin, 138(2), 322. https://doi.org/10.1037/a0026744

Menganathan, R. (2011). Language policy in education and the role of English in India: From library language to language of empowerment. In H. Coleman (Ed.), Dreams and realities: Developing countries and the English language. British Council. https://www.teachingenglish.org.uk/sites/teach eng/files/Z413\%20EDB\%20Section04_0.pdf

Metsala, J. L., \& Walley, A. C. (1998). Spoken vocabulary growth and the segmental restructuring of lexical representations: Precursors to phonemic awareness and early reading ability. In J. L. Metsala \& L. C. Ehri (Eds.), Word recognition in beginning literacy (pp. 89-120). Lawrence Erlbaum Associates Publishers. 
Mishra, R., \& Stainthorp, R. (2007). The relationship between phonological awareness and word reading accuracy in Oriya and English: A study of Oriya-speaking fifth-graders. Journal of Research in Reading, 30(1), 23-37. https://doi.org/10.1111/j.1467-9817.2006.00326.x

Nag, S. (2007). Early reading in Kannada: The pace of acquisition of orthographic knowledge and phonemic awareness. Journal of Research in Reading, 30(1), 7-22. https://doi.org/10.1111/j.1467-9817. 2006.00329.x

Nag, S. (2011). The Akshara languages: What do they tell us about children's literacy learning? In R. Mishra \& N. Srinivasan (Eds.), Language-cognition: State of the art (pp. 291-310). Lincom.

Nag, S., \& Snowling, M. J. (2012). Reading in an alphasyllabary: Implications for a language-universal theory of learning to read. Scientific Studies of Reading, 16, 404-423. https://doi.org/10.1080/10888 438.2011.576352

Nakamura, P. R., de Hoop, T., \& Holla, C. (2019). Language and the learning crisis: Evidence of transfer threshold mechanisms in multilingual reading in South India. The Journal of Development Studies, 55(11), 2287-2305. https://doi.org/10.1080/00220388.2018.1493192

Nakamura, P. R., Joshi, R. M., \& Ji, X. R. (2018). Investigating the asymmetrical roles of syllabic and phonemic awareness in Akshara processing. Journal of Learning Disabilities, 51(5), 499-506. https://doi.org/10.1177/0022219417718201

Nakamura, P. R., Koda, K., \& Joshi, R. M. (2014). Biliteracy acquisition in Kannada and English: A developmental study. Writing Systems Research, 6(1), 132-147. https://doi.org/10.1080/17586801. 2013.855620

Nimon, K., Oswald, F., \& Roberts, J. K. (2020). Yhat: Interpreting regression effects. R Package Version 2.0-3. Available at https://cran.r-project.org/web/packages/yhat/yhat.pdf

Nimon, K. (2010). Regression commonality analysis: Demonstration of an SPSS solution. Multiple Linear Regression Viewpoints, 36(1), 10-17.

Nishanimut, S. P., Johnston, R. S., Joshi, R. M., Thomas, P. J., \& Padakannaya, P. (2013). Effect of synthetic phonics instruction on literacy skills in an ESL setting. Learning and Individual Differences, 27, 47-53. https://doi.org/10.1016/j.lindif.2013.06.007

Office of the Registrar General and Census Commissioner India. (2011). Census of India: Language. https://censusindia.gov.in/2011Census/C-16_25062018_NEW.pdf

Patel, P., Torppa, M., Aro, M., Richardson, U., \& Lyytinen, H. (2021). Assessing the effectiveness of a game-based phonics intervention for first and second grade English language learners in India: A randomized controlled trial. Journal of Computer Assisted Learning, 38(1), 76-89. https://doi.org/ $10.1111 /$ jcal.12592.

Perfetti, C. A. (2003). The universal grammar of reading. Scientific Studies of Reading, 7(1), 3-24. https://doi.org/10.1207/S1532799XSSR0701_02

Perfetti, C. A., Liu, Y., \& Tan, L. H. (2005). The lexical constituency model: Some implications of research on Chinese for general theories of reading. Psychological Review, 112(1), 43-59. https:// doi.org/10.1037/0033-295X.112.1.43

Pufpaff, L. A. (2009). A developmental continuum of phonological sensitivity skills. Psychology in the Schools, 46(7), 679-691. https://doi.org/10.1002/pits.20407

Rao, C., Sumathi, T. A., Midha, R., Oberoi, G., Kar, B., Khan, M., Vaidya, K., Midya, V., Raman, N., Gajre, M., \& Singh, N. C. (2021). Development and standardization of the DALI-DAB (Dyslexia Assessment for Languages of India-Dyslexia Assessment Battery). Annals of Dyslexia. https://doi. org/10.1007/s11881-021-00227-z

Reddy, P. P., \& Koda, K. (2013). Orthographic constraints on phonological awareness in biliteracy development. Writing Systems Research, 5(1), 110-130. https://doi.org/10.1080/17586801.2012.748639

Scarborough, H. S. (2005). Developmental relationships between language and reading: Reconciling a beautiful hypothesis with some ugly facts. In H. W. Catts \& A. G. Kamhi (Eds.), The connections between language and reading disabilities (pp. 3-24). Erlbaum.

Share, D. L., \& Daniels, P. T. (2016). Aksharas, alphasyllabaries, abugidas, alphabets and orthographic depth: Reflections on Rimzhim, Katz and Fowler (2014). Writing Systems Research, 8(1), 17-31. https://doi.org/10.1080/17586801.2015.1016395

Simons, G. F., \& Fennig, C. D. (Eds.). (2018). Ethnologue: Languages of the world (21st ed.). SIL International.

Singh, N. C. (2015). Dyslexia Assessment for Languages of India (DALI). National Brain Research Centre. 
Singh, N. C., \& Sumathi, T. A. (2019). The role of phonological processing and oral language in the acquisition of reading skills in Devanagari. In R. M. Joshi \& C. McBride (Eds.), Handbook of literacy in Akshara orthography (pp. 261-276). Springer.

Thompson, B. (2006). Foundations of behavioral statistics: An insight-based approach. Guilford Press.

Treiman, R., \& Zukowski, A. (1991). Levels of phonological awareness. In S. A. Brady \& D. P. Shankweiler (Eds.), Phonological processes in literacy: A tribute to Isabelle Y. Liberman (pp. 67-83). Lawrence Erlbaum Associates Inc.

Vaid, J., \& Gupta, A. (2002). Exploring word recognition in a semi-alphabetic script: The case of Devanagari. Brain and Language, 81(1-3), 679-690. https://doi.org/10.1006/brln.2001.2556

Vishnoi, A. (2020, March 30). No switch in instruction medium from English to regional languages with NEP '20: HRD. The Economic Times. https://economictimes.indiatimes.com/industry/services/ education/no-switch-in-instruction-medium-from-english-to-mother-tongue/regional-languageswith-nep-20/articleshow/77271164.cms?from $=$ mdr

Wagner, R. K., \& Torgesen, J. K. (1987). The nature of phonological processing and its causal role in the acquisition of reading skills. Psychological Bulletin, 101(2), 192-212. https://doi.org/10.1037/ 0033-2909.101.2.192

Wang, M., Park, Y., \& Lee, K. R. (2006). Korean-English biliteracy acquisition: Cross-language phonological and orthographic transfer. Journal of Educational Psychology, 98(1), 148-158. https://doi. org/10.1037/0022-0663.98.1.148

Wawire, B. A., \& Kim, Y.-S.G. (2018). Cross-Language transfer of phonological awareness and letter knowledge: Causal evidence and nature of transfer. Scientific Studies of Reading, 22(6), 443-461. https://doi.org/10.1080/10888438.2018.1474882

Ziegler, J., \& Goswami, U. (2005). Reading acquisition, developmental dyslexia and skilled reading across languages: A psycholinguistic grain size theory. Psychological Bulletin, 31(1), 3-29. https:// doi.org/10.1037/0033-2909.131.1.3

Ziegler, J. C., \& Goswami, U. (2006). Becoming literate in different languages: Similar problems, different solutions. Developmental Science, 9(5), 429-436. https://doi.org/10.1111/j.1467-7687.2006. 00509.x

Publisher's Note Springer Nature remains neutral with regard to jurisdictional claims in published maps and institutional affiliations. 